ONLINE MUTATION REPORT

\title{
Loss of five amino acids in BRCA2 is associated with ovarian cancer
}

\author{
S L Martinez, J Herzog, J N Weitzel
}

J Med Genet 2004;41:e18 (http://www.jmedgenet.com/cgi/content/full/41/2/e18). doi: 10.1136/jmg.2003.010827

$\mathrm{H}$ ereditary breast and ovarian cancer (HBOC) syndrome is characterised by an early age of onset and an autosomal dominant pattern of inheritance. Mutations in BRCA1 or BRCA2 account for the majority of families with HBOC, with carriers bearing a lifetime risk of approximately $50-80 \%$ for breast cancer and $15-45 \%$ for ovarian cancer. ${ }^{12}$ Most deleterious mutations are small insertions/deletions, nonsense mutations, or splice site mutations that result in a frame shift and/or premature protein truncation. However, minor alterations such as missense mutations or small in frame insertions/deletions in the coding regions of BRCAl and $B R C A 2$ are often the only change detected. These present a clinical dilemma in cancer risk counselling because of their classification as variants of unknown significance (VUS), which are essentially uninformative findings. ${ }^{3}$

A number of analytical strategies exist to elucidate the significance of a VUS. If the variant has been reported previously, the commercial vendor of the gene test provides ancillary data about the VUS, such as the number of observations, predominant racial or ethnic origin, whether seen concurrently with a known deleterious mutation, and whether the VUS tracks with disease in families where such testing is possible. In addition, given the presumed tumour suppressor function of the BRCA proteins, it has been suggested that somatic allelic deletion of the wild type allele in a tumour, as detected by loss of heterozygosity ( $\mathrm{LOH})$ analysis, can provide evidence for the deleterious nature of the respective germline mutations. ${ }^{4}$ Finally, the degree of evolutionary conservation of the amino acid sequence and the presence of functional domains in a given region of the protein can be considered circumstantial evidence for the importance of the amino acids encompassing that domain. ${ }^{5}$

BRCA gene sequencing (Myriad Genetic Laboratories Inc, Salt Lake City, UT, USA) of a 50 year old ovarian cancer patient from an HBOC family revealed a previously unreported $15 \mathrm{bp}$ in frame deletion in exon 18 of $B R C A 2$ (8457del15), classified as a VUS. This mutation is predicted to result in the deletion of five amino acids (2744-2748; RLTVG) in the carboxyterminal region of the protein. We report here studies to elucidate the clinical significance of this mutation.

\section{METHODS}

Personal and family history, blood samples, and archival tumours were obtained from the patient and first degree relatives after written informed consent for participation in an institutional review board approved prospective hereditary cancer registry (IRB\#96144). To investigate whether the mutation tracked with disease in the family, we isolated leukocyte DNA from the proband, two unaffected sisters, and a daughter, using a salting out method (AGTC; Denver, CO, USA). Paraffin embedded ovarian tumour tissue blocks from the proband and her deceased mother (history of breast cancer at 57 years of age and ovarian cancer at 62 years) were sectioned and microdissected to yield material with $>90 \%$ tumour or normal cells, and DNA was extracted using a

\section{Key points}

- Mutations in BRCA1 and BRCA2 are responsible for the majority of families with hereditary breast and ovarian cancer (HBOC) syndrome, and most deleterious mutations are small insertions/deletions, nonsense mutations, or splice site mutations that result in a truncated protein.

- The significance of minor alterations in coding regions such as missense mutations and small in frame insertions/deletions frequently cannot be inferred, so they are classified as variants of unknown significance, which creates a clinical dilemma in genetic counselling.

- We confirmed the probable clinical significance of a $15 \mathrm{bp}$ in frame deletion in exon 18 of BRCA2 (8457del15) that was discovered through BRCA gene sequencing of a 50 year old ovarian cancer patient from an HBOC family.

- The variant was found to track exclusively in affected family members. Tumour samples from two carriers showed selective loss of the wild type allele at the BRCA2 locus, and the extent of chromosomal loss determined by $\mathrm{LOH}$ was distinct for each tumour. The region of BRCA2 harbouring the mutation revealed high evolutionary conservation across species and two overlapping functional domains that may be disrupted in carriers of this variant.

standard phenol/chloroform procedure following digestion with proteinase K. Exon 18 of BRCA2 was amplified using published primer sequences and a modified touchdown PCR program. Nested primers encompassing the 8457dell5 mutation were designed to amplify a $161 \mathrm{bp}$ fragment from the initial PCR product (forward: 5'CAGATGgGtgGtATGCTGTtAAGG and reverse: 5'CATAAGAGATTCTGGGGCTTCAAG). This $161 \mathrm{bp}$ amplicon was then used for $\mathrm{LOH}$ experiments and for sequencing on an ABI 377 automated sequencer (Applied Biosystems; Foster, CA, USA). A single PCR amplification (using only the inner primers) was sufficient for DNA extracted from whole blood.

Six microsatellite markers spanning chromosome 13 (D13S742, D13S218, D13S171, D13S267, D13S263, and D13S248; listed in physical map order) were amplified from matched normal and tumour sample pairs using published primer sequences. The markers were also amplified in

Abbreviations: $\mathrm{HBOC}$, hereditary breast and ovarian cancer; $\mathrm{LOH}$, loss of heterozygosity; OB, oligonucleotide/oligosaccharide binding; VUS, variant of unknown significance 
unaffected relatives to gain haplotype information to determine the phase of the wild type allele. PCR products were analysed by PAGE on $8 \%(30 \mathrm{~cm} \times 40 \mathrm{~cm}, 0.8 \mathrm{~mm}$ spacers) gels in $1 \times \mathrm{TBE}$ and visualised by ethidium bromide staining. $\mathrm{LOH}$ was determined semi-quantitatively $(>50 \%$ allelic imbalance), and loci were scored as uninformative (homozygous in normal tissue), informative (heterozygous), or LOH.

Analysis of evolutionary conservation for BRCA2 was performed by a multisequence alignment using the SeqWeb comparison program Pile-Up (Accelrys; San Diego, CA, USA). Protein sequences were obtained by linking out to "Protein Neighbors" from human BRCA2 on the NCBI website (www.ncbi.nlm.nih.gov). We conducted a PubMed (National Library of Medicine) search and reviewed published manuscripts reporting on functional and structural studies of BRCA2.

\section{RESULTS}

This was the first report of the BRCA2 (8457del15) VUS by the vendor, therefore no ancillary data were available. Sequencing of the PCR product from the affected mother's tumour block confirmed the presence of the $15 \mathrm{bp}$ deletion (fig 1). The smaller, mutated allele was clearly detected in both the proband and her mother by electrophoresis of the $161 \mathrm{bp}$ fragment on a native $8 \%$ acrylamide gel. Conversely, the unaffected siblings and daughter of the proband were all negative for the mutation (fig 2). The probability of the variant tracking solely with disease in this family (that is, carrier status of the five individuals, and their respective affected/unaffected status) $v$ chance (the 50:50 odds of inheriting a specific autosomal allele) is $1 / 32$ (0.03). Excluding the 24 year old daughter, the probability is $1 / 16$ (0.06), although the current ages of the other unaffected members may not be adequate to exclude potential ovarian cancer expression (the age at onset is not reliably earlier in $B R C A$ carriers). In addition, tumours from both the proband and her mother showed loss of the wild type allele compared with normal tissue (fig 2C).

Analysis of six microsatellite markers along chromosome 13 revealed selective loss of the wild type allele in both the proband and her mother (data not shown). In the proband's tumour, all informative markers revealed $\mathrm{LOH}$ including distal markers on either end of the chromosome, indicating that complete somatic loss of chromosome 13 may have occurred. The mother's tumour was uninformative for the $13 p$ terminal marker (D13S742) and retained heterozygosity at D13S218. All other markers analysed displayed LOH. Thus, the somatic loss in each tumour was unique.

An alignment of BRCA2 amino acid sequences from five species (human, mouse, rat, dog, and chicken) indicated significant evolutionary conservation in the region, with 39/ 50 amino acid identity (78\%) between mammals (human,

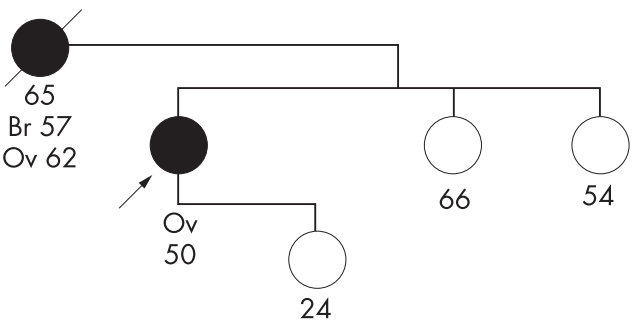

B
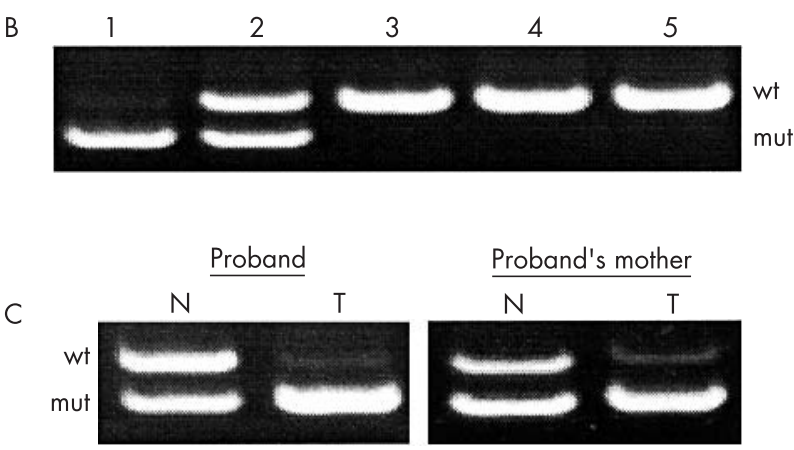

Figure 2 Segregation of the 8457 del 15 mutation in the family. (A) Abbreviated pedigree of the family with ages and affected status (filled symbols) indicated. (B) The PCR product from the section of exon 18 encompassing the mutation was analysed by PAGE for each individual. Lane 1 is from a tumour sample from the proband's deceased mother. Only the mutant allele is represented owing to the selective loss of the wild type allele. Lane 2, the proband, depicts the presence of the wild type and mutated allele in genomic DNA. Lane 3 is the proband's daughter, and lanes 4 and 5 are the unaffected siblings of the proband, all of whom are apparently homozygous for the wild type allele. (C) Comparison of normal $v$ tumour DNA for both the proband and her mother at the 8457 del 15 locus reveal loss of heterozygosity, with selective loss of the wild type allele in each case.

mouse, rat, and dog), including four of the five amino acids involved in the deletion (fig 3). A conservative change, from arginine to lysine, was seen at amino acid position 2744 in mouse. All other residues are identical across the five species including chicken.

Finally, a comprehensive PubMed search was conducted to collect information on mutations occurring within the region of interest, as well as any pertinent structural data. Of note, examination of recent crystallographic data from the BRCA2 carboxyterminus ${ }^{6}$ revealed that in addition to being located within one of three oligonucleotide/oligosaccharide binding (OB) folds, the 8457dell5 variant obliterates three DSS1 contacting residues.

\section{DISCUSSION}

VUS in the BRCA genes continue to be problematic in cancer risk counselling. If the VUS tracks exclusively with disease in

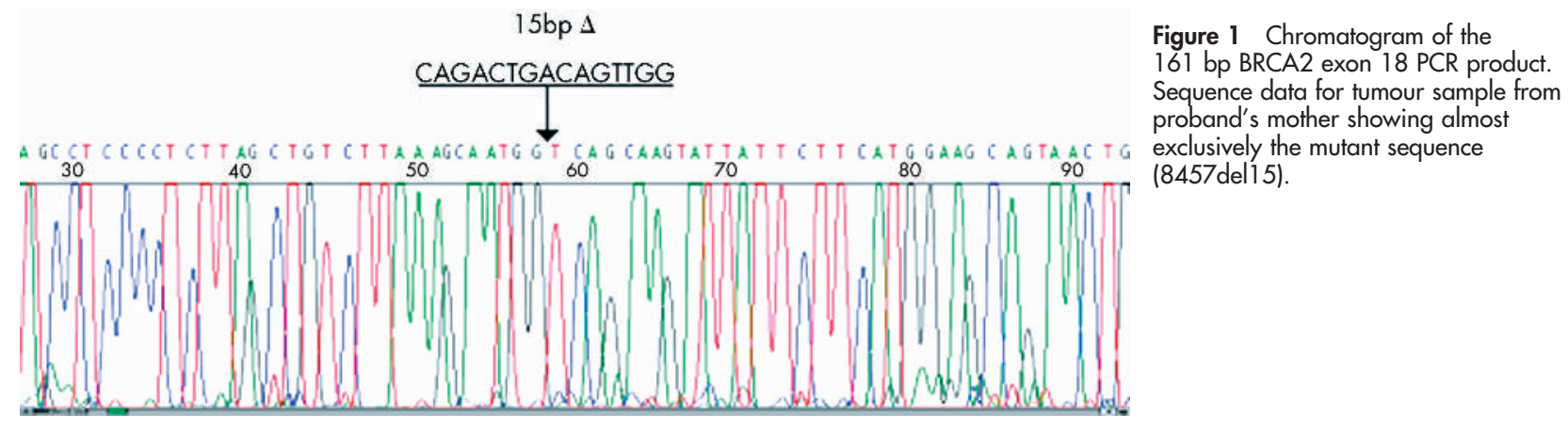

Figure 1 Chromatogram of the 161 bp BRCA2 exon 18 PCR product. 作 s most exclusively the mutant sequence (8457del15). 
2690

$\begin{array}{llll}\text { Human } & \text { VSDIISLSAN } & \text { ISETSSNKTS } & \text { SADTQKVAII } \\ \text { Mouse } & \text { ISDIISPSTK } & \text { VSETSGGKTS } & \text { GEDANKVDTI } \\ \text { Rat } & \text { VSDIISLSTN } & \text { VSETSGSKAS } & \text { SEDSNKVDTI } \\ \text { Dog } & \text { ISEIISSSAD } & \text { ISETSSSKTS } & \text { SVGTKKVGII } \\ \text { Chicken } & \text { VSKVLSLNTA } & \text { VSPSNSN.NN } & \text { TEGEKAAAII }\end{array}$

2740

Human LKNG $\overline{R L T V} G Q$ KIILHGAELV

Mouse VKSGKLTVGQ KIITQGAELV

Rat VKSGRLTVGQ KIITQGAELV

Dog VKNGRLTVGQ KITIHGAELV

Chicken LHRRRLTVGQ KIIVHGAELI
2739

ELTDGWYAVK AQLDPPLLAV

ELTDGWYAVR AQLDPPLMAL

ELTDGWYAVK AQLDPPLLAL

ALTDGWYAIK AQLDPPLLAL

EVTDGWYGIR ALLDPPLKAF

2789

SPDACTPLE APESLMLKIS ASTRPARWY

SPDACAPLE APDSLRLKIS ASTRPARWH

SPDACAPLE APDSLRLKIS ASTRPARWH

SPDACTPLE APESLMLKIS ASTRPACWY

SPNGCTPLE APDSLMLKIA ASTRCARWY
Figure 3 Multisequence alignment of BRCA2 orthologs. The five amino acids deleted in 8457del 15 (2744-2748), depicted by the solid black bar, are highly conserved with only a conservative change from arginine to lysine in the first amino acid position in mouse, in addition to high conservation across the region, with 39/50 amino acid identity. the family, as it does here, the likelihood of pathogenicity is increased. However, studies of tracking within a single family are seldom definitive because of limited statistical power, phenocopies, and incomplete age dependent penetrance. The strength of the association can be augmented by informative LOH studies demonstrating loss of the wild type allele in tumours. Furthermore, microsatellite markers can be useful for determining the extent of the somatic chromosomal loss. The use of sequence databases to determine the degree of evolutionary conservation in the amino acid sequence region in which the variant occurs is a complementary approach. High conservation indicates probable biological significance of the region in relation to protein function.

The 8457 dell 15 variant reported here has not previously been observed in published literature or in the Breast Cancer Information Core database. Our analysis indicated that the mutation tracked exclusively with the affected members in this family, and that there was somatic allelic deletion of the wild type allele in each case. This mutation is predicted to result in the deletion of five of the 3418 amino acids that constitute the BRCA2 protein, and lies within the highly conserved carboxyterminal domain. Conservation of the five specific amino acids seen here indicates that the region harbouring the deletion may have functional significance.

With respect to known functional domains, 8457del15 lies well downstream of the ovarian cancer cluster region ${ }^{8}$ and the BRC repeats (Rad 51 binding sites). Recent crystallographic data ${ }^{6}$ indicates that this variant results in the loss of three DSSI contacting residues. Although DSS1 has not been linked to carcinogenesis to date, it may function by stabilizing BRCA2 within the cell or have some other as yet undefined role in DNA repair. Crystallography has also revealed that the 8457 dell 15 variant lies within one of three OB folds, which are present in most single stranded DNA binding proteins. Consequently, disruption of this OB fold by the variant could compromise BRCA2 binding to ssDNA during homologous recombination.

Based on the facts that the BRCA2 8457del15 variant tracks exclusively with affected family members, with concurrent

\section{ELECTRONIC DATA ACCESS}

Breast Cancer Information Core (accession number 4993): http://www.nhgri.nih.gov/Intramural_research/ Lab_transfer/Bic/ PubMed: http://www.ncbi.nlm.nih.gov./PubMed/ Online Mendelian Inheritance in Man (OMIM). BRCA] (OMIM 113705) and BRCA2 (OMIM600185). http://www. ncbi.nlm.nih.gov/Omim/ loss of the wild type allele in tumours from both affected carriers, coupled with the data on evolutionary conservation and possible disruption of functional domains, we conclude that the $15 \mathrm{bp}$ deletion is highly likely to be deleterious. This information has clinical relevance for at risk members of this family and others who may have alterations affecting this region of BRCA2.

\section{ACKNOWLEDGEMENTS}

This study was supported in part by the California Research Program (grant no. 99-86874). We wish to thank Dr G Schlake for assistance with microdissections and Dr C Szabo and Dr T Frank for helpful discussions.

\section{Authors' affiliations}

S L Martinez, J Herzog, J N Weitzel, Department of Clinical Cancer Genetics, Beckman Research Institute

J N Weitzel, City of Hope (COH) Comprehensive Cancer Center, Duarte, CA, USA

Correspondence to: Dr J N Weitzel, Director, Department of Clinical Cancer Genetics, City of Hope Cancer Center, 1500 E Duarte Road, Duarte, CA 91010, USA; jweitzel@coh.org

\section{REFERENCES}

1 Ford D, Easton DF, Stratton M, Narod S, Goldgar D, Devilee P, Bishop DT, Weber B, Lenoir G, Chang-Claude J, Sobol H, Teare MD, Struewing J, Arason A, Scherneck S, Peto J, Rebbeck TR, Tonin P, Neuhausen S, Barkardottir R, Eyfjord J, Lynch H, Ponder BAJ, Gayther SA, Birch JM, Lindblom BA, Stoppa-Lyonnet D, Bignon Y, Borg A, Hamann U, Haites N, Scott RJ, Maugard CM, Vasen H, Seitz S, Cannon-Albright LA, Schofield A, Zelada-Hedman M. Genetic heterogeneity and penetrance analysis of the BRCA1 and BRCA2 genes in breast cancer families. The Breast Cancer Linkage Consortium. Am J Hum Genet 1998;62:676-89.

2 Struewing JP, Hartge P, Wacholder S, Baker SM, Berlin M, McAdams M, Timmerman MM, Brody LC, Tucker MA. The risk of cancer associated with specific mutations of BRCA1 and BRCA2 among Ashkenazi Jews. NEngl J Med 1997:336:1401-8.

3 Weitzel JN. Genetic cancer risk assessment: Putting it all together. Cancer 1999;86:2483-92.

4 Osorio A, de la Hoya M, Rodriguez-Lopez R, Martinez-Ramirez A, Cazorla A, Granizo JJ, Esteller M, Rivas C, Caldes T, Benitez J. Loss of heterozygosity analysis at the BRCA loci in tumor samples from patients with familial breast cancer. Int J Cancer 2002;99:305-9.

5 Welcsh PL, Owens KN, King MC. Insights into the functions of BRCA1 and BRCA2. Trends Genet 2000;16:69-74.

6 Yang $\mathbf{H}$, Jeffrey PD, Miller J, Kinnucan E, Sun Y, Thoma NH, Zheng $N$, Chen PL, Lee WH, Pavletich NP. BRCA2 function in DNA binding and recombination from a BRCA2-DSS1-ssDNA structure. Science 2002;297: 1837-48

7 Greenblatt MS, Beaudet JG, Gump JR, Godin KS, Trombley L, Koh J, Bond JP. Detailed computational study of p53 and pl6: using evolutionary sequence analysis and disease-associated mutations to predict the functional consequences of allelic variants. Oncogene 2003;22:1150-63.

8 Neuhausen SL, Godwin AK, Gershoni-Baruch R, Schubert E, Garber J, Stoppa-Lyonnet D, Olah E, Csokay B, Serova O, Lalloo F, Osorio A Stratton M, Offit K, Boyd J, Caligo MA, Scott RJ, Schofield A, Teugels E, Schwab M, Cannon-Albright L, Bishop T, Easton D, Benitez J, King M-C, Ponder BAJ, Weber B, Devilee P, Borg A, Narod SA, Goldgar D. Haplotype and phenotype analysis of nine recurrent BRCA2 mutations in 111 families: results of an international study. Am J Hum Genet 1998;62:1381-8. 